\title{
Social and Economic Factors and Malnutrition or the Risk of Malnutrition in the Elderly: A Systematic Review and Meta-Analysis of Observational Studies
}

\author{
Maria Besora-Moreno ${ }^{1}$, Elisabet Llauradó ${ }^{1, *(\mathbb{D})}$, Lucia Tarro ${ }^{1,2}$ and Rosa Solà ${ }^{1,2,3}$ \\ 1 Universitat Rovira i Virgili, Facultat de Medicina i Ciències de la Salut, Functional Nutrition, Oxidation, and \\ Cardiovascular Diseases Group (NFOC-Salut), 43201 Reus, Spain; mariadelaserra.besora@urv.cat (M.B.-M.); \\ lucia.tarro@urv.cat (L.T.); rosa.sola@urv.cat (R.S.) \\ 2 Centre Tecnològic de Catalunya, Unitat de Nutrició i Salut, Eurecat, 43204 Reus, Spain \\ 3 Hospital Universitari Sant Joan de Reus, Department of Internal Medicine, 43204 Reus, Spain \\ * Correspondence: elisabet.llaurado@urv.cat; Tel.: +34-977-75-89-20
}

Received: 20 February 2020; Accepted: 8 March 2020; Published: 11 March 2020

\begin{abstract}
Malnutrition in the elderly could be tackled by addressing socioeconomic factors. This study aimed to determine the magnitude of the relationship between socioeconomic factors and the malnutrition or malnutrition risk (MR) in the elderly. The PubMed and SCOPUS databases were searched for observational studies that included assessment of malnutrition or/and MR and socioeconomic variables (educational level, living alone, marital status, income and occupational level, feeling of loneliness, place of residence, and food expenditure) in $\geq 60$-year-old subjects, published in English among 2000-2018 (PROSPERO: CRD42019137097). The systematic review included 40 observational studies ( 34 cross-sectional and 4 cohort studies) and 16 cross-sectional studies in the meta-analysis (34,703 individuals) of malnutrition and MR in relation to low educational level (Odds Ratio (OR): 1.48; 95\% Confidence Interval (CI): 1.33-1.64; $p<0.001$ ), living alone (OR: 1.92; 95\% CI: 1.73-2.14; $p<0.001$ ), being single, widowed, or divorced (OR: 1.73; 95\% CI: 1.57-1.90; $p<$ 0.001 ), and low income level (OR: 2.69; 95\% CI: 2.35-3.08; $p<0.001$ ), and considering these four socioeconomic factors, malnutrition and MR is associated with them (OR: 1.83; 95\% CI: $1.73-1.93 ; p$ $<0.001)$. Malnutrition and MR could be reduced by increasing economic level, supporting people living alone or being single, widowed, and divorced, and improving lifelong learning.
\end{abstract}

Keywords: malnutrition; nutritional status; elderly; socioeconomic factors

\section{Introduction}

Nowadays, the aging of the population is a consequence of the increase in life expectancy [1]. In Europe, between 2015 and 2050, the proportion of the world's population over 60 years will nearly double from $12 \%$ to $22 \%$, with a different distribution of gender and by country [2].

Aging can be associated with malnutrition, which is a public health problem characterized by a multifactorial physiological state [3,4]. Malnutrition is defined as an insufficient nutritional intake or absorption, which leads to a decrease in fat and muscle mass [5,6]. From the European Society for Clinical Nutrition and Metabolism (ESPEN) definition, malnutrition is diagnosed by a body mass index (BMI) of $<18.5 \mathrm{~kg} / \mathrm{m}^{2}$ or by meeting two of these three criteria: unintentional weight loss $(>10 \%$ in an indefinite time period or $>5 \%$ over the last three months) combined with either a low BMI (BMI of $<20 \mathrm{~kg} / \mathrm{m}^{2}$ if $<70$ years of age, or $<22 \mathrm{~kg} / \mathrm{m}^{2}$ if $\geq 70$ years of age) or a low fat-free mass index (FFMI) score (FFMI of $<15$ and $<17 \mathrm{~kg} / \mathrm{m}^{2}$ in women and men, respectively) [7]. The overall prevalence of malnutrition in the elderly ranges from $1 \%$ to $24.6 \%$ [8]. In addition, $50 \%$ of the elderly in rehabilitation, 
$20 \%$ in residential care, and $40 \%$ in hospitals are malnourished [9]. As a result of population aging, the malnutrition prevalence is increasing, which is expected to reach $29.1 \%$ by 2080 [1].

However, before reaching the need of a malnutrition diagnosis, the use of validated tools could be used to determine malnutrition risk [6,7]. According to the ESPEN, the Mini Nutritional Assessment (MNA) is the most effective tool for screening and evaluating the risk of malnutrition in the elderly $[6,10,11]$, composed of four areas related to anthropometry, clinical status (illness, medications, psychological stress, neuropsychological problems), dietary assessment, and self-perception about health and nutrition [10]. Other tools used to assess the risk of malnutrition include the Malnutrition Screening Tool (MUST) and the Nutrition Risk Screening (NRS), but these do not include the evaluation of important malnutrition risk factors such as functional, psychological, and cognitive parameters [10].

Consequently, a state of malnutrition can cause an impairment of quality of life, especially in the elderly, and can lead to increased healthcare costs and hospital stays [12].

Additionally, malnutrition is related to health, environmental, and social factors or determinants [6]. The elderly are more vulnerable to developing a worse nutritional status because of their lack of teeth, their loss of taste, or their poor mobility [5]; as a consequence, these factors can affect dietary intake. The social factors associated with malnutrition include lifestyle, loneliness, isolation, marital status, educational level, socioeconomic level, and place of residence $[3,6]$. Those that are single, widowed, or divorced are the most malnourished or at the most risk of malnutrition, especially the widowed [13]. The elderly with low educational levels have worse nutritional status due to the lack of cooking skills or insufficient knowledge to make healthy food choices [14]. Furthermore, there is a higher prevalence of malnutrition in people who live in rural areas [8]. Another determinant of malnutrition is income level, with a strong relationship between the levels of poverty and malnutrition in the elderly [13]. Thus, the social and economic determinants of malnutrition risk and/or malnutrition development should be analyzed to establish which determinants affect malnutrition and in which magnitude, as a basis for taking possible actions to reduce malnutrition in the elderly.

Thus, the hypothesis was that social and economic factors-such as low educational level, living alone, being single, widowed, or divorced, low income level, low previous (before 60 years old) or current occupational level, feeling of loneliness, living in rural areas, and low food expenditure-are related to malnutrition and malnutrition risk in the elderly.

The main objective of the present systematic review and meta-analysis was to assess and determine the magnitude of the relationship between socioeconomic factors and the malnutrition or malnutrition risk in the elderly $\geq 60$ years old.

\section{Materials and Methods}

We performed a systematic review and meta-analysis of observational studies regarding malnutrition and malnutrition risk and their relation to socioeconomic factors in the elderly. This study was reported in accordance with the Preferred Reporting Items for Systematic Reviews and Meta-Analyses (PRISMA) (Table S1) and it was registered in PROSPERO International Prospective Register of Systematic Reviews (CRD42019137097).

\subsection{Search Strategy}

An exhaustive literature search in the PubMed and SCOPUS electronic databases was carried out, as these databases are considered the largest and the most multidisciplinary, covering medical and social sciences journals that include the principal outcomes of the present meta-analysis (malnutrition and socioeconomic factors). Depending on the study, socioeconomic factors were assessed separately as social factors and economic factors. Regarding nutritional status, some studies assessed the malnutrition and the malnutrition risk separately, while other studies evaluated them together. For this reason, the search was done using the following keywords combined with each other: malnutrition, nutritional status, older, elderly, social factors, social, economic, and socioeconomic. The search strategies in PubMed were: "older" or "elderly" and "social" and "malnutrition", "older" or "elderly" 
and "economic" and "malnutrition", "nutritional status" and "social factors"; and in SCOPUS were: "economic" and "malnutrition" and "older" or "elderly", "social" and "malnutrition" and "older" or "elderly".

\subsection{Study Selection}

The inclusion criteria were observational studies (case-control, prospective cohort, and cross-sectional studies), with the target population $\geq 60$ years old, in which publications with data on malnutrition status, as well as social and economic variables, were published in English between January 2000 and December 2018.

The studies that did not meet all of the inclusion criteria were excluded. Additionally, studies that only included populations with illness or comorbidities were also excluded in order to focus on the general population, who was not selected taking into account a specific disease, to be able to generalize the results.

\subsection{Article Review and Data Extraction}

The search strategy of the studies that matched the inclusion criteria was carried out with the online platform Covidence (Covidence systematic review software, Veritas Health Innovation, Melbourne, Australia; available at www.covidence.org). Firstly, the studies were selected based on the title information. Subsequently, the abstracts of these selected studies were checked to see if they met or not the inclusion and exclusion criteria. Finally, those studies that met the inclusion criteria were full-text assessed. When the studies did not have all of the information necessary to be included in the present study, an email was sent to the authors of the publication requesting this information. The data extraction was carried out independently. Of the included studies, the following variables were collected: authors, year of study development, the country of implementation, the type of study, number of participants, target population's age, gender, social factors (educational level, living alone or cohabit, marital status, feeling of loneliness, place of residence), economic factors (income level, occupational level, food expenditure), nutritional status screening tool, nutritional status (risk of malnutrition, malnutrition, and optimal nutritional status), and study quality. The previous or current occupational level was divided into low occupational levels (such as farmer, breeder, housewife, laborer, or hard manual or physical work) and high occupational levels (such as employee, businessman, worker, administration, or jobs involving higher intellectual effort).

\subsection{Risk of Bias in Individual Studies}

To facilitate the quality assessment of the present study and to minimize the risk of bias in the included studies, the Quality Assessment Tool for Observational Cohort and Cross-Sectional Studies [15] was used with the following criteria: the cross-sectional studies were evaluated on 10 points, because questions $6,7,12$, and 13 are only for cohort studies, while the prospective cohort studies were evaluated on 14 points. Observational studies were divided into three quality categories (high, medium, and low) with their respective cut-off points: (a) for cross-sectional studies, quality categories were: low quality ( $\leq 3$ points), medium quality ( $4-7$ points), and high quality (8-10 points); and (b) for prospective cohort studies, quality categories were: low quality ( $\leq 5$ points), medium quality (6-9 points), and high quality (10-14 points).

\subsection{Statistical Analysis: Meta-Analysis}

To carry out the meta-analysis, we used the Review Manager (RevMan) (computer program: version 5.3. Copenhagen: The Nordic Cochrane Centre, The Cochrane Collaboration, 2014). To select studies for the meta-analysis, it was taken into account whether they used the MNA as a nutritional evaluation tool (as this was the most used tool to evaluate the nutritional status in the elderly among the studies) and evaluated the socioeconomic variable with the same criteria. To include a study, information about the percentage or number of subjects with a risk of malnutrition or 
malnutrition-related to the socioeconomic variable was required. In contrast, studies that assessed all outcomes via multivariate analysis were excluded from the meta-analysis. Thus, we only performed the meta-analysis of each socioeconomic factor, in which at least 4 studies evaluated this factor and followed the inclusion criteria described previously. In addition, an analysis was performed in general (all variables in one) and by a subgroup of the same variables. To evaluate the heterogeneity of the studies, we used the $\mathrm{I}^{2}$ statistic. When the heterogeneity was over $85 \%$, they were analyzed with randomized and non-fixed effects. A $p$-value of $<0.05$ was considered statistically significant.

\section{Results}

A total of 363 studies were obtained from the literature search, of which 60 were eliminated by duplicity. Of the remaining 303 studies, 197 were excluded according to the inclusion and exclusion criteria based on the information provided in the title and the abstract. A total of 106 studies were full-text assessed, and 66 were excluded because of the following reasons: results not related to the outcomes studied $(n=48)$, text not available $(n=8)$, non-representative population sample $(n=5)$, and wrong study design $(n=5)$. Finally, as shown in Figure 1, 40 studies were included in the systematic review [13,14,16-53] and 16 studies in the meta-analysis $[13,20,21,25,26,29,30,35,36,39,42,43,45,51-53]$.

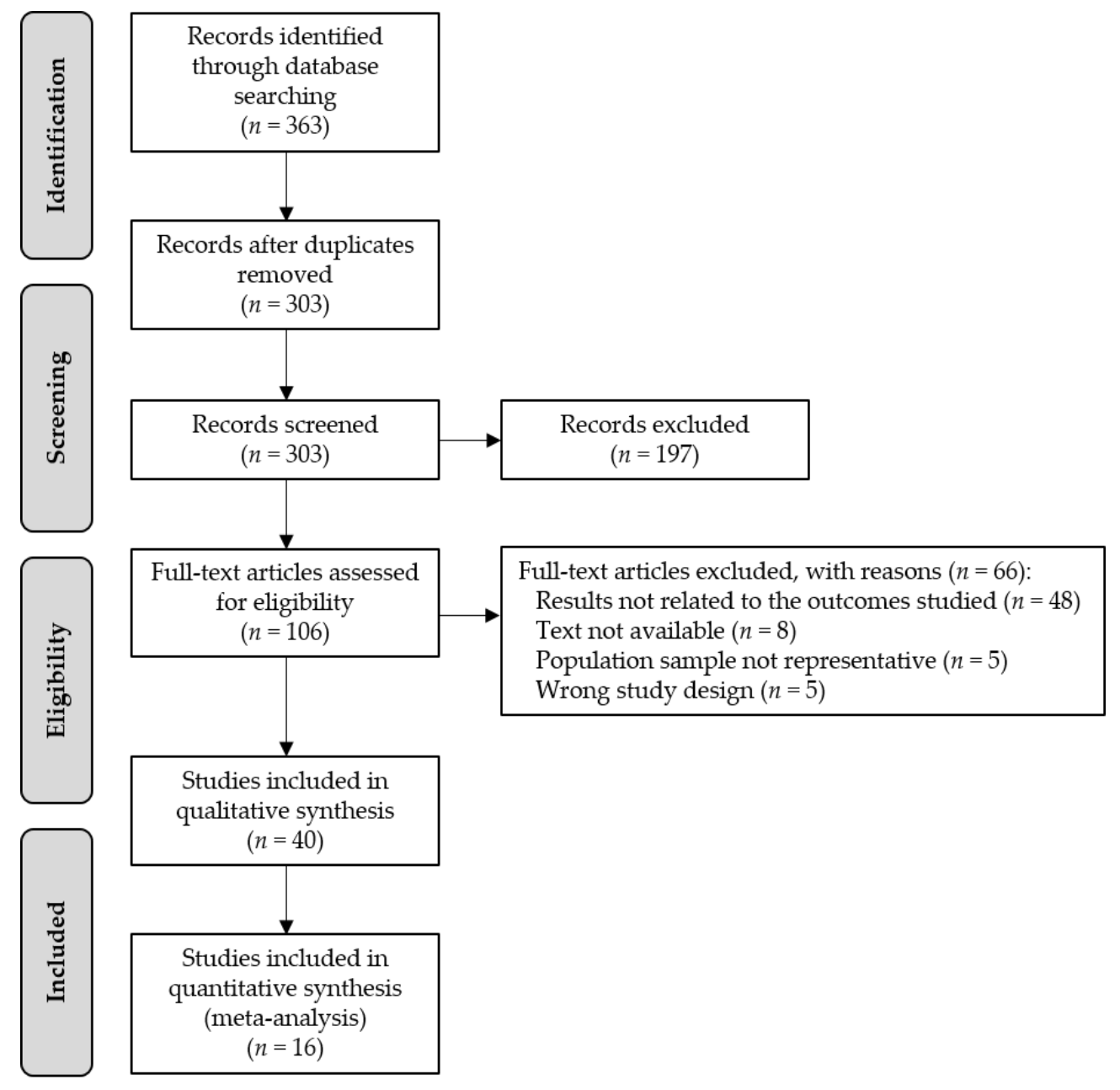

Figure 1. Preferred Reporting Items for Systematic Reviews and Meta-Analyses (PRISMA) flow diagram of the study selection. 


\subsection{Characteristics of the Included Studies in the Systematic Review}

Of the total 40 included studies, 36 were cross-sectional $[13,14,17,18,20-32,34-49,51-53]$ and four were cohort studies $[16,19,33,50]$. The study population was 60 years old and above, except for two studies that focused only on people aged over either 85 years [26] or 90 years [37]. The total number of participants in the different studies ranged from 67 subjects [48] to 15,669 subjects [49], with a total of 61,818 subjects included in the present systematic review. All of the studies included people of both genders, except two studies [50,51] — one of them was only focused on men [50] and the other only on women [51]. Of the 40 studies included in the systematic review, according to the Development Human Index from the United Nations Development Program (UNDP) [54], 33 were conducted in regions with a high development human index (Poland [39], Sweden [38,40], China [30,37,53], Turkey [52], Canada [49,50], Italy [14,35], Taiwan [16], Lebanon [17,20,25], Brazil [18], Ireland [19,31], Finland [21], Spain [13,22,26], Norway [23,34], Portugal [24], Korea [27], Iran [28], Sri Lanka [29], New Zealand [48], Mexico [32], Amsterdam [33], Malaysia [36], and Shanghai [51]), five were conducted in regions with a medium development human index (Bangladesh [41,44], India [42], South Africa [43], and Egypt [45]), and two were conducted in regions with a low development human index (Central Africa [46] and Nigeria [47]).

Of the 40 observational studies included in the systematic review, regarding the tools used to assess the nutritional status, 29 studies used the MNA [13,14,16-18,20,21,23-26,28-32,34-37,39-43,45,51-53], one of which also used the Norwegian version of the Nutritional Form For the Elderly (NUFFE-NO) [34]. Of the other studies, five used only the Body Mass Index (BMI) [19,22,33,44,46] and one also used self-reported weight loss [19], four used the Senior in the community: risk evaluation for eating and nutrition (SCREEN II) [38,48-50] and two used the Nutritional Screening Initiative (NSI) [27,47]. Only three studies considered depression as a pathology added to malnutrition $[18,28,43]$.

Of the total of 40 observational studies included in the systematic review, eight different socioeconomic variables were evaluated: 31 studies evaluated the educational level $[13,14,16,18-20$, 22,24-30,33,35-37,39,41-49,51-53], 27 the fact of living alone or cohabiting [13,14,19,21,22,25-29,31,32, 36-40,42-45,48-53], 24 the marital status [13,14,16,19,23,24,26,27,29,30,33-36,39,41,42,44-46,48,51-53], 20 the income level $[14,16,20,22,24,25,27,29,30,33,35,37,39,41-45,47,51,52], 12$ the occupational level $[14,28,30,34-37,44-46,51,53]$, six the feeling of loneliness $[13,17,21,33,34,36]$, three the place of residence [28,39,51], and one the food expenditure [41] (Table 1).

Table 1. Quality assessment and socioeconomic variables analyzed of the observational studies included in the systematic review.

\begin{tabular}{|c|c|c|}
\hline & Quality & Socioeconomic Variables Analyzed \\
\hline Bardon et al., 2018 [19] & Medium & Educational level, living alone, marital status \\
\hline Boulos et al., 2016 [17] & High & Feeling of loneliness \\
\hline Cabrera et al., 2007 [18] & Medium & Educational level \\
\hline Chen et al., 2007 [16] & Medium & Educational level, marital status, income level \\
\hline Damayanthi et al., 2018 [29] & High & Educational level, living alone, marital status, income level \\
\hline Debnath et al., 2017 [44] & Medium & $\begin{array}{c}\text { Educational level, living alone, marital status, income level, } \\
\text { occupational level }\end{array}$ \\
\hline Donini et al., 2013 [14] & Medium & $\begin{array}{l}\text { Educational level, living alone, marital status, income level, } \\
\text { occupational level }\end{array}$ \\
\hline El Zoghbi et al., 2014 [20] & Medium & Educational level, income level \\
\hline El-Desouky et al., 2018 [45] & High & $\begin{array}{l}\text { Educational level, living alone, marital status, income level, } \\
\text { occupational level }\end{array}$ \\
\hline Eskelinen et al., 2016 [21] & Medium & Living alone, feeling of loneliness \\
\hline Farre et al., 2014 [26] & High & Educational level, living alone, marital status \\
\hline Ferdous et al., 2009 [41] & High & Educational level, marital status, income level, food expenditure \\
\hline
\end{tabular}


Table 1. Cont.

\begin{tabular}{|c|c|c|}
\hline & Quality & Socioeconomic Variables Analyzed \\
\hline Ferra et al., 2012 [22] & High & Educational level, living alone, income level \\
\hline Fjell et al., 2018 [23] & Medium & Marital status \\
\hline Gündüz et al., 2015 [52] & Medium & Educational level, living alone, marital status \\
\hline Jésus et al., 2017 [46] & High & $\begin{array}{l}\text { Educational level, marital status, } \\
\text { occupational level }\end{array}$ \\
\hline Ji et al., 2012 [37] & Medium & Educational level, living alone, income level, occupational level \\
\hline Johansson et al., 2009 [40] & Medium & Living alone \\
\hline Jun et al., 2016 [51] & High & $\begin{array}{l}\text { Educational level, living alone, marital status, income level, } \\
\text { occupational level, place of residence }\end{array}$ \\
\hline Krzymińska-Siemaszko et al., 2014 [39] & Medium & $\begin{array}{l}\text { Educational level, living alone, marital status, income level, place of } \\
\text { residence }\end{array}$ \\
\hline Lengyel et al., 2017 [50] & Medium & Living alone \\
\hline Lin et al., 2016 [53] & High & Educational level, living alone, marital status, occupational level \\
\hline Madeira et al., 2018 [24] & High & Educational level, marital status, income level \\
\hline Maseda et al., 2017 [13] & Medium & Educational level, living alone, marital status, feeling of loneliness \\
\hline Mathew et al., 2017 [42] & Medium & Educational level, living alone, marital status, income level \\
\hline Mitri et al., 2016 [25] & High & Educational level, living alone, income level \\
\hline Mokhber et al., 2011 [28] & Low & Educational level, living alone, occupational level, place of residence \\
\hline Naidoo et al., 2015 [43] & High & Educational level, living alone, income level \\
\hline Olayiwola et al., 2006 [47] & Medium & Educational level, income level \\
\hline Park et al., 2014 [27] & Medium & Educational level, living alone, marital status, income level \\
\hline Ramage-Morin et al., 2013 [49] & Medium & Educational level, living alone \\
\hline Rodriguez-Tadeo et al., 2011 [32] & Medium & Living alone \\
\hline Romero-Ortuno et al., 2010 [31] & Medium & Living alone \\
\hline Schilp et al., 2011 [33] & High & Educational level, marital status, income level, loneliness feeling \\
\hline Shi et al., 2014 [30] & High & Educational level, marital status, income level, occupational level \\
\hline Söderhamn et al., 2012 [34] & Medium & $\begin{array}{l}\text { Marital status, occupational level, } \\
\text { feeling of loneliness }\end{array}$ \\
\hline Suzana et al., 2013 [36] & Medium & $\begin{array}{l}\text { Educational level, living alone, marital status, occupational level, } \\
\text { feeling of loneliness }\end{array}$ \\
\hline Timpini et al., 2011 [35] & Medium & Educational level, marital status, income level, occupational level \\
\hline Westergren et al., 2014 [38] & High & Living alone \\
\hline Wham et al., 2015 [48] & High & Educational level, living alone, marital status \\
\hline
\end{tabular}

\subsection{Educational Level and Malnutrition/Malnutrition Risk}

Of the 31 studies that evaluated educational level, 15 established significant relationships with regard to malnutrition or malnutrition risk $[14,22,25,27,28,35,39,41,42,45-47,49,52,53]$, two suggested a significant trend $[30,44]$, and 14 did not present significant differences $[13,16,18-20,24,26,29,33,36$, $37,43,48,51]$ (Table S2). Of those 15 with signification, 11 studies showed a negative association, that is, the lower the educational level, the higher the malnutrition risk $[14,25,28,35,39,41,42,45,46,49,53]$. Five of these evaluated the Odds Ratio (OR) [35,42,45,46,49], revealing an increased malnutrition risk of between 1.3 and 8 times more in those who had low educational levels or fewer years of schooling $[35,42,45,46,49]$. In addition, one study presented results with the $\beta$ coefficient [41], showing that the highest educational level was significantly associated with the greatest score in the MNA questionnaire [41]. Instead, the results of four studies were significantly contrary. On the one hand, two studies established that a low educational level protected against malnutrition [22,52]—one of which even related a low educational level with higher overweight and obesity risk [22]. On the other hand, one of the other studies indicated that the elderly with fewer years of schooling had better 
nutritional profiles [27], while the other study established a positive relationship between educational level and malnutrition according to the Pearson correlation [47].

\subsection{Living Alone or Cohabiting and Malnutrition/Malnutrition Risk}

A total of 27 studies evaluated the relationship between living alone or cohabiting and the malnutrition or malnutrition risk, of which 13 showed statistically significant results $[13,14,21,27,28$, 32,38,39,45,49-51,53] and 14 showed no significance [19,22,25,26,29,31,36,37,40,42-44,48,52] (Table S3). Of those 13 studies with statistically significant results, 11 showed that the elderly who lived alone had better malnutrition than the elderly who cohabited with other people-whether the spouse, sons, grandsons, or other family or friends [13,14,21,27,28,32,38,39,49,50,53]. Two of these evaluated the OR [32,38] and one of them also evaluated the $\beta$ coefficient [32]. An increased risk of malnutrition was demonstrated in the elderly who lived alone [32,38], and it was observed that living alone increased malnutrition risk by 1.8 times [32]. In addition, the increased risk was found both in men and women who lived alone [38]. One study found that there was a higher significant percentage of people living with a spouse who had a better nutritional status [13]. However, two studies with significant results showed contrary results, establishing that of those with malnutrition, there was a higher percentage who lived together $[45,51]$.

\subsection{Marital Status and Malnutrition/Malnutrition Risk}

A total of 24 studies evaluated the relation between the marital status and malnutrition or malnutrition risk, of which 11 showed significant results $[14,19,27,30,33-35,39,42,45,53]$ and 13 were not significant [13,16,23,24,26,29,36,41,44,46,48,51,52] (Table S4). Of those 11 studies with significant results, 10 showed that the elderly who were single, widowed, or divorced had a greater malnutrition risk than those who were married $[14,19,27,33-35,39,42,45,53]$. Only one study showed significance in relation to people with malnutrition who were in day centers, pointing out that a significantly higher percentage of these were widows [14]. A further four evaluated the OR [19,34,42,45] and one the Hazard Ratio (HR) [33]. All of the studies that evaluated the OR concluded that the risk of malnutrition was 1.64 times higher in single or divorced elderly [19], 2.19 times higher in single, divorced, or widowed people [42], 2.99 times higher in single elderly [34], and 29.4 times higher in divorced or widowed elderly [45]. HR also established that not having a partner was associated with a higher malnutrition risk [33]. Only one study obtained significant results in contrast to those defined above, expressing that of those elderly with a worse nutritional status, there was a higher percentage who were married [30].

\subsection{Income Level and Malnutrition/Malnutrition Risk}

Of the total of 20 studies that evaluated the income level, 13 established significant relationships [14,22,24,25,27,35,39,41-43,45,47,51], one suggested a significant trend [44], and six did not present significant differences [16,20,29,30,33,37] (Table S5). Of those 13 studies with significant results, 12 suggested that a low income level was associated with a higher risk of malnutrition $[14,22,24,25,27,35,39,41-43,45,51]$. Of these 12 studies with a negative association, seven evaluated the OR $[22,24,35,42,43,45,51]$ and three the $\beta$ coefficient $[41,43,51]$. The OR of five studies determined that the malnutrition risk ranged between 1.31 [22] and 64.7 [45] times more in the elderly with low income levels. In another study, the OR showed that the elderly with low socioeconomic status had 6.01 times more risk of malnutrition [42]. One study concluded that having income and receiving finance on a regular basis is significantly associated with an increase in the score in the MNA questionnaire [41]. Lastly, it was found that a high economic level protects against malnutrition [51]. In contrast, however, from the significant studies, one study determined that at a high-income level there are higher levels of malnutrition [47]. 


\subsection{Occupational Level and Malnutrition/Malnutrition Risk}

Of the total of 12 studies that evaluated the previous ( $<60$ years old) or current occupational level, six obtained statistically significant results $[14,28,34,45,46,53]$ and six did not $[30,35-37,44,51]$ (Table S6). Of the studies with statistical significance, four showed that people with low current occupational levels or who were retired or unemployed had a higher percentage of malnutrition or malnutrition risk $[14,28,34,45]$. Two of these evaluated the OR [34,45] and one of them the $\beta$ coefficient [34]. One study established that unemployed people had 22.2 times more risk of malnutrition [45]. In another study, the OR and the $\beta$ coefficient revealed that administrative professions protect against malnutrition [34]. The OR of the other significant study showed that working ( $<60$ years old or currently) as a farmer or animal breeder increased the risk of malnutrition [46]. Only one study obtained significant opposite results, expressing a high percentage of malnutrition among working elderly people [53].

\subsection{Feeling of Loneliness and Malnutrition/Malnutrition Risk}

Of the total six studies that evaluated the feeling of loneliness, four were related significantly with malnutrition or malnutrition risk [13,17,21,33], one suggested a significant trend [34], and only one did not show significant results [36] (Table S7). Of the significant studies, two resolved that the elderly with a greater feeling of loneliness had a higher prevalence of malnutrition $[17,33]$. Of these studies, one evaluated the HR [33], showing an increased risk of malnutrition of 1.47 in the elderly who had feelings of loneliness. In contrast, two studies had significant opposite results, showing that the elderly with a lower score in the MNA questionnaire (malnutrition) did not express feelings of loneliness $[13,21]$.

\subsection{Place of Residence and Malnutrition/Malnutrition Risk}

A total of three studies evaluated the place of residence, which was differentiated between urban and rural. Only one study established significant relationships with malnutrition or malnutrition risk [28] and two did not show significant results [39,51] (Table S8). The only study with significant results showed that the elderly who lived in rural areas had worse nutritional status and more malnutrition or malnutrition risk, in comparison with those who lived in urban areas [28].

\subsection{Food Expenditure and Malnutrition/Malnutrition Risk}

Only one study evaluated the relation between food expenditure and malnutrition or malnutrition risk, and it did not show statistically significant results [41] (Table S9).

\subsection{Quality of the Articles Included in the Systematic Review}

Of the total 40 studies included, 16 were of high quality $[17,22,24-26,29,30,33,38,41,43,45,46,48$, $51,53], 23$ of medium quality [13,14,16,18-21,23,27,31,32,34-37,39,40,42,44,47,49,50,52], and one of low quality [28]. Of the 16 studies of high quality, one of them was a cohort study [33]. Of the 23 studies of medium quality, three of them were cohort studies $[16,19,50]$. Regarding the questionnaire, questions $1,2,9,11$, and 14, which refer to the research question, study population, exposure measures, and assessment, outcome measures, and statistical analyses, respectively, were the most reported by the studies. In contrast, questions 5 and 10, which refer to the sample size justification and the repeated exposure assessment, respectively, were the least reported by the studies. The quality of the studies is shown in Table 1 and Table S10.

\subsection{Meta-Analysis}

Of the total of 40 studies included in the systematic review, 16 cross-sectional studies were included in the meta-analysis $[13,20,21,25,26,29,30,35,36,39,42,43,45,51-53]$. The meta-analysis included a sample of 34,703 individuals, of which 10,755 people had or were at risk of malnutrition and 23,948 had good nutritional status; the Forest Plot is shown in Figure 2. In general, evaluating all variables at once, it 
was observed that having a low educational level, living alone, being single, widowed, or divorced, and having a low income level increased malnutrition and the risk of malnutrition in the elderly (OR: 1.83; 95\% Confidence Interval (CI): 1.73-1.93; $p<0.001 ; \mathrm{I}^{2}=94 \% ; p$ about heterogeneity $<0.001$ ) (Figure 2). The 16 cross-sectional studies included in the meta-analysis were analyzed by subgroups depending on the socioeconomic variable $[13,20,21,25,26,29,30,35,36,39,42,43,45,51-53]$.

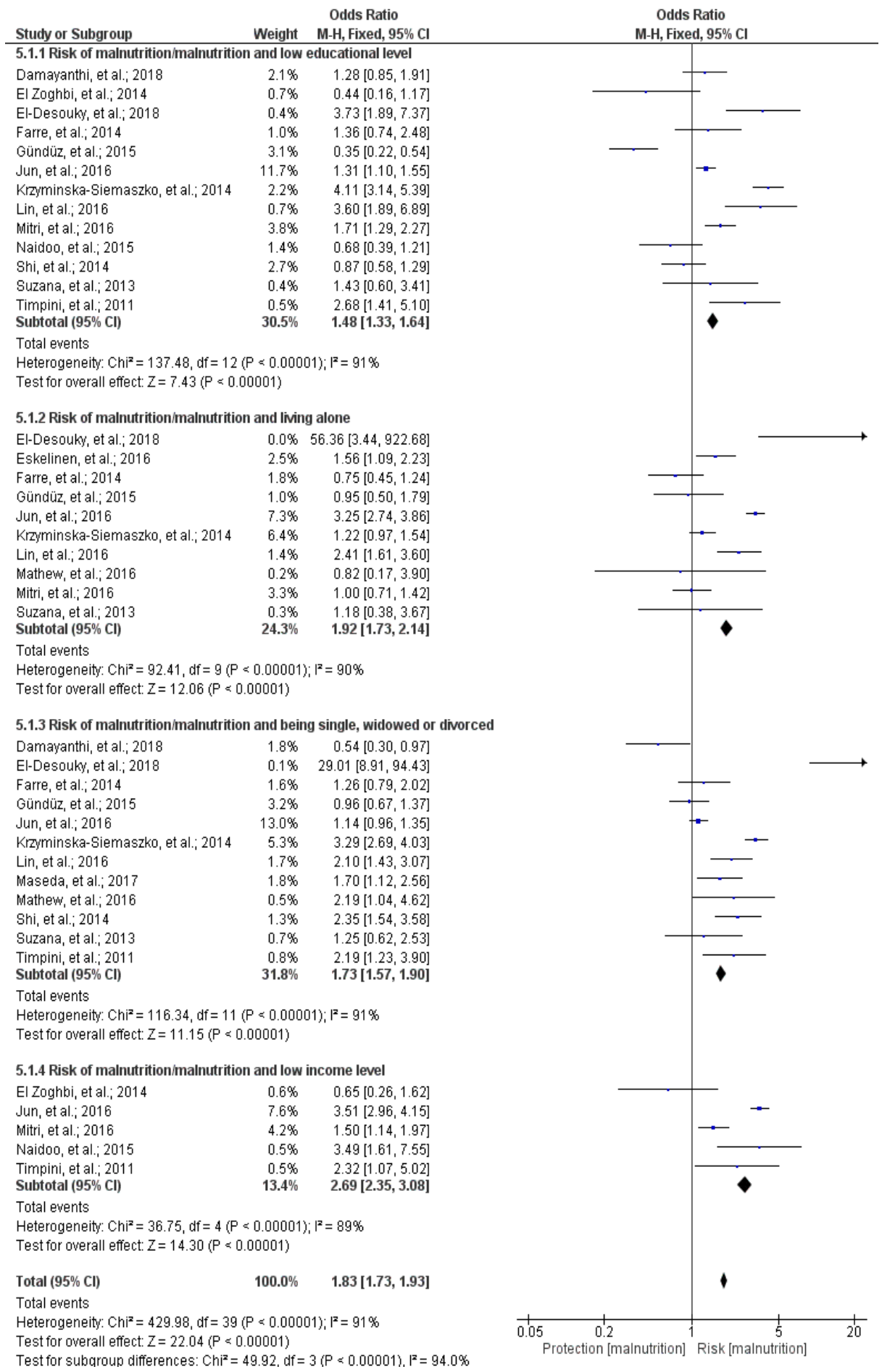

Figure 2. Forest plot of the relationship between malnutrition and/or malnutrition risk and socioeconomic factors (low educational level, living alone, marital status of single, widowed, or divorced, and low income level) individually and by subgroups, in alphabetical order, in the elderly (aged $\geq 60$ years). 


\subsubsection{Educational Level and Malnutrition/Malnutrition Risk}

Thirteen studies that evaluated the educational level in relation to malnutrition or malnutrition risk were included $[20,25,26,29,30,35,36,39,43,45,51-53]$. This meta-analysis included a sample of 10,734 individuals, of which 3233 had or were at risk of malnutrition. It was observed that having a low educational level is a risk factor for malnutrition (OR: $1.48 ; 95 \%$ CI: $1.33-1.64 ; p<0.001 ; \mathrm{I}^{2}=91 \% ; p$ about heterogeneity < 0.001) (Figure 2).

\subsubsection{Living Alone or Cohabiting and Malnutrition/Malnutrition Risk}

Ten studies that evaluated the relationship between living alone and malnutrition or malnutrition risk were included $[21,25,26,36,39,42,45,51-53]$. This meta-analysis included a sample of 9042 individuals, of which 3034 had or were at risk of malnutrition. It was observed that living alone is a risk factor for malnutrition (OR: 1.92; 95\% CI: 1.73-2.14; $p<0.001 ; \mathrm{I}^{2}=90 \% ; p$ about heterogeneity < 0.001) (Figure 2).

\subsubsection{Marital Status and Malnutrition/Malnutrition Risk}

Twelve studies that evaluated the marital status and malnutrition or malnutrition risk were included [13,26,29,30,35,36,39,42,45,51-53]. This meta-analysis included a sample of 10,083 individuals, of which 2843 had or were at risk of malnutrition. It was observed that being single, widowed, or divorced (in comparison to being married) is a risk factor for malnutrition (OR: 1.73; 95\% CI: 1.57-1.90; $p<0.001 ; \mathrm{I}^{2}=91 \% ; p$ about heterogeneity $<0.001$ ) (Figure 2).

\subsubsection{Income Level and Malnutrition/Malnutrition Risk}

Five studies that evaluated the income level and malnutrition or malnutrition risk were included [20,25,35,43,51]. This meta-analysis included a sample of 4844 individuals, of which 1645 had or were at risk of malnutrition. It was observed that having a low income level is a risk factor for malnutrition (OR: 2.69; 95\% CI: 2.35-3.08; $p<0.001 ; \mathrm{I}^{2}=89 \%$; $p$ about heterogeneity $<0.001$ ) (Figure 2).

\section{Discussion}

The present systematic review and meta-analysis of observational studies, which were mainly cross-sectional, supports the hypothesis that social and economic factors are related to malnutrition and malnutrition risk in people aged $\geq 60$ years. Focused on the meta-analysis outcomes, according to the socioeconomic factor, in a descendant order, low income level, living alone, being single, widowed, or divorced, and low educational level had a greater relationship with malnutrition and malnutrition risk in the elderly. Meanwhile, there were not enough articles providing scientific evidence to make a meta-analysis of some of the other socioeconomic factors, such as low occupational level (e.g., farmer, breeder, housewife, laborer, or hard manual or physical work), feeling of loneliness, living in rural areas, and food expenditure. Thus, it was not possible to prove whether these factors act as a malnutrition risk factor in the elderly.

In the current meta-analysis, it was observed that low educational level is a risk factor for malnutrition, suggesting the predisposition to malnutrition. A possible explanation is that individuals with a higher educational level have more knowledge about nutrition and health [55], leading to a healthier and more varied diet and, therefore, a better nutritional status than individuals with a low educational level.

Another identified risk factor from the results of the present meta-analysis is living alone, which showed a significant association with the risk of developing malnutrition. The elderly who lived alone had a greater malnutrition risk compared to others who cohabit with someone-either family, acquaintances, or friends. Three literary reviews [56-58] obtained the same results as the present review and related the fact of living alone with malnutrition. Moreover, gender can influence the risk of malnutrition. A review of the literature observed that men who lived with their wives had 
better nutrition than men who lived alone, because their wives were in charge of regularly cooking and grocery shopping [59]. Another explanation is that the elderly who lived alone had a worse nutritional status due to the lack of social interaction with other people [55]. More specifically, in a study investigating the effect of the presence of others, both within the household and during meals, on caloric intake in homebound older adults, it was concluded that the elderly who ate with other people consumed more calories than those who ate alone [60]. Thus, a simple and inexpensive way to increase caloric intake is to make arrangements for family members or caregivers to eat with them [60]. In contrast to the results obtained in the present meta-analysis, in two systematic reviews of the determinants of malnutrition, living alone was not identified as a risk factor for malnutrition $[4,61]$.

In reference to marital status, the present meta-analysis established an association between being single, widowed, and divorced and the risk of malnutrition. In the same way to the present results, one review concluded that widowed individuals, independently of gender, were more vulnerable to the risk of nutritional deficiencies [62]. The possible explanations could be that, firstly, widowhood is associated with poor eating habits and with less enjoyment of eating [63], for example, the loss of the social interaction culturally related to food. Secondly, the wife's death implies a deterioration in the husband's nutritional status as a consequence of inexperience in food tasks in this generation [59]. Despite the evidence shown so far, one systematic review established that the death of a spouse was not associated with malnutrition in the elderly [4].

Furthermore, the present meta-analysis also established an association between lowest income levels and a higher risk of developing malnutrition in the elderly. One explanation is based on the fact that the healthiest or freshest food is the most expensive, so are not accessible for the elderly with few economic resources [64]. Thus, all of these low income-associated aspects could increase the risk of malnutrition and could be a determinant of malnutrition in the elderly.

Another socioeconomic factor evaluated is the low previous ( $<60$ years old) or current occupational level, namely, farmer, breeder, housewife, laborer, hard manual or physical work, or unemployment, which were identified, in the present systematic review, as a malnutrition risk factor. However, there were not enough articles to perform a meta-analysis. A possible explanation is that those elderly with a low occupational level usually have a lower economic level too, so they might not have access to high-quality nutritional food.

Regarding the feeling of loneliness, which is separate from social isolation, the present systematic review obtained low numbers of articles relative to the risk of malnutrition. Only two cross-sectional studies showed that the elderly with a greater feeling of loneliness were at greater risk of malnutrition $[17,33]$, while other reviews did not find this association $[57,62,65,66]$. One review stated that the aging process was related to psychosocial and environmental changes, such as loneliness [65]. These changes could have a negative effect on nutritional status [65]. Probably, the feeling of loneliness in the elderly causes a loss of appetite and interest in meals. The feeling of loneliness, along with isolation and decreased social interactions, worsens the nutritional vulnerability in the elderly [62]. On the contrary, one systematic review of prospective studies suggested that the feeling of loneliness was not related to the risk of malnutrition [61].

The present meta-analysis could not establish a reliable association regarding the place of residence, either urban or rural, and malnutrition risk in the elderly. Of all of the studies included in the review, only one cross-sectional study showed that people in Iran who lived in rural areas had a greater risk of malnutrition [28]. In the same way, a cross-sectional study concluded that the MNA score was significantly lower in people who lived in rural areas compared to others who lived in urban areas, while the rural elderly had lower educational and income levels than the urban elderly [67]. As mentioned earlier, low education and low income levels could negatively affect their nutritional status [67]. Finally, another socioeconomic factor that could not establish a reliable association with malnutrition in this systematic review and meta-analysis was that of food expenditure, probably related to the economic level of the elderly. As stated above, a low economic level means not having enough money for food [68]. 
This systematic review and meta-analysis presents different limitations. First of all, the present systematic review was about observational studies, mainly cross-sectionals. Future studies should evaluate the influence of socioeconomic factors in malnutrition of randomized controlled intervention studies to obtain maximum evidence [69]. The second limitation was that not all studies used a unified definition to evaluate malnutrition or malnutrition risk. For example, from the studies included in the meta-analysis, seven evaluated malnutrition $[20,29,39,42,43,52,53]$, two evaluated only the risk of malnutrition [35,36], and seven evaluated malnutrition and risk of malnutrition at the same time $[13,21,25,26,30,45,51]$. It should be clarified in future studies the distinction between malnutrition and risk of malnutrition to improve the quality of the evidence. In addition, studies either did not use the same tool to evaluate socioeconomic factors, or the tool used was not described appropriately. Thirdly, few studies evaluated the feeling of loneliness, the place of residence, or the food expenditure; therefore, no meta-analysis could be done on how these socioeconomic factors were associated with malnutrition and malnutrition risk. In addition, specifically on the occupational level, the jobs analyzed did not represent all professions. Fourthly, socioeconomic psychosocial factors, such as loneliness, isolation, or depression need to be studied, since they have an important burden in the development of malnutrition in the elderly [62]. The last limitation is that when performing the meta-analysis, a high level of heterogeneity of the included studies was observed, which represents an important limitation [70]. This heterogeneity could be due to the inclusion of the general population (illness and not illness individuals), as it excluded studies that only included ill people.

All of the results of the present systematic review and meta-analysis serve to better understand the social and economic risk factors associated with malnutrition in the elderly $\geq 60$ years old. Moreover, the identification of social and economic risk factors allows us to avoid them, and thus to reduce or prevent malnutrition or/and malnutrition risk and to maintain a good nutritional status of the elderly. Thus, regarding the evidence provided in the present systematic review and meta-analysis, the following table shows a proposal of actions on socioeconomic risk factors to reduce or prevent malnutrition and malnutrition risk. These actions can provide a basis for future nutritional interventions on socioeconomic factors to diminish malnutrition in the elderly. However, more research is needed to confirm that these interventions are effective in combating each socioeconomic factor (Table 2).

Table 2. Proposal of nutritional interventions to prevent malnutrition and malnutrition risk based on the socioeconomic factors presented in elderly.

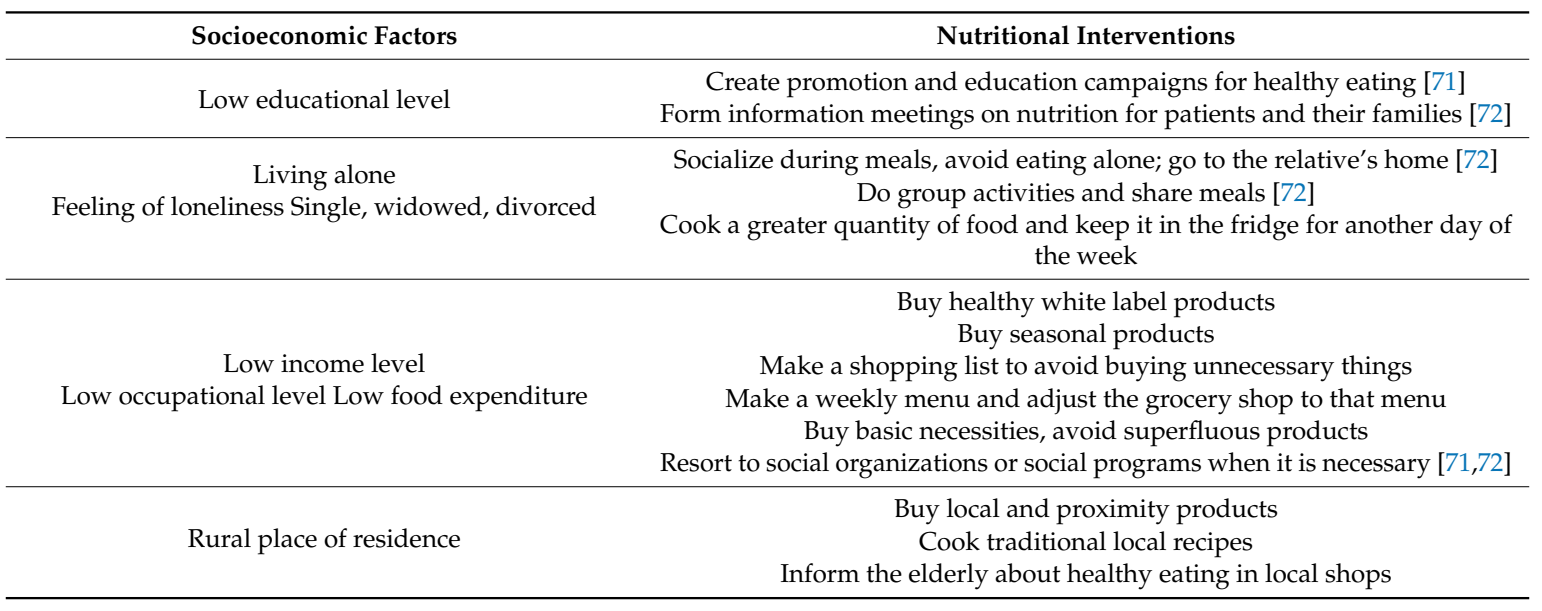

\section{Conclusions}

Malnutrition and malnutrition risk could be reduced in the elderly by increasing their economic level, supporting those living alone or who are single, widowed, or divorced, and improving lifelong learning. 
Supplementary Materials: The following are available online at http://www.mdpi.com/2072-6643/12/3/737/s1, Table S1: Preferred Reporting Items for Systematic Reviews and Meta-Analyses (PRISMA) criteria; Table S2: Characteristics of included studies about the relationship between educational level and malnutrition and/or malnutrition risk; Table S3: Characteristics of included studies about the relationship between living alone or not and malnutrition and/or malnutrition risk; Table S4: Characteristics of included studies about the relationship between marital status and malnutrition and/or malnutrition risk; Table S5: Characteristics of included studies about the relationship between income level and malnutrition and/or malnutrition risk; Table S6: Characteristics of included studies about the relationship between occupational level and malnutrition and/or malnutrition risk; Table S7: Characteristics of included studies about the relationship between feeling of loneliness and malnutrition and/or malnutrition risk; Table S8: Characteristics of included studies about the relationship between place of residence and malnutrition and/or malnutrition risk; Table S9: Characteristics of included studies about the relationship between food expenditure and malnutrition and/or malnutrition risk; Table S10: Quality assessment of the observational studies included in systematic review.

Author Contributions: Conceptualization, M.B.-M., E.L., L.T., and R.S.; methodology, M.B.-M., E.L., L.T., and R.S.; formal analysis, M.B.-M., E.L., L.T., and R.S.; investigation, M.B.-M., E.L., L.T., and R.S.; writing-Original draft preparation, M.B.-M., E.L., L.T., and R.S.; writing-review and editing, M.B.-M., E.L., L.T., and R.S.; supervision, M.B.-M., E.L., L.T., and R.S.; project administration, M.B.-M., E.L., L.T., and R.S.; All authors have read and agreed to the published version of the manuscript.

Funding: This research received no external funding.

Conflicts of Interest: The authors declare no conflicts of interest.

\section{References}

1. European Commission Population Structure and Ageing. Available online: https://ec.europa.eu/eurostat/ stati-sticsexplained/index.php/Population_structure_and_ageing\#Past_and_future_population_ageing_ trends_in_the_EU (accessed on 9 December 2019).

2. World Health Organization Ageing and Health. Available online: https://www.who.int/news-room/factsheets/detail/ageing-and-health (accessed on 8 January 2020).

3. Streicher, M.; Zwienen-Pot, J.; Bardon, L.; Nagel, G.; Teh, R.; Meisinger, C.; Colombo, M.; Torbahn, G.; Kiesswetter, E.; Flechtner-Mors, M.; et al. Determinants of incident malnutrition in community-dwelling older adults: A MaNuEL multicohort meta-analysis. J. Am. Geriatr. Soc. 2018, 66, 2335-2343. [CrossRef] [PubMed]

4. Van der Pols-Vijlbrief, R.; Wijnhoven, H.A.H.; Schaap, L.A.; Terwee, C.B.; Visser, M. Determinants of protein-energy malnutrition in community-dwelling older adults: A systematic review of observational studies. Ageing Res. Rev. 2014, 18, 112-131. [CrossRef] [PubMed]

5. Hickson, M. Malnutrition and ageing. Postgrad. Med. J. 2006, 82, 2-8. [CrossRef] [PubMed]

6. Corish, C.A.; Bardon, L.A. Malnutrition in older adults: Screening and determinants. Proc. Nutr. Soc. 2018, 78, 372-379. [CrossRef] [PubMed]

7. Cederholm, T.; Bosaeus, I.; Barazzoni, R.; Bauer, J.; Van Gossum, A.; Klek, S.; Muscaritoli, M.; Nyulasi, I.; Ockenga, J.; Schneider, S.M.; et al. Diagnostic criteria for malnutrition-An ESPEN Consensus Statement. Clin. Nutr. 2015, 34, 335-340. [CrossRef]

8. Crichton, M.; Craven, D.; Mackay, H.; Marx, W.; de van der Schueren, M.; Marshall, S. A systematic review, meta-analysis and meta-regression of the prevalence of protein-energy malnutrition: Associations with geographical region and sex. Age Ageing 2018, 48, 38-48. [CrossRef]

9. Kaiser, M.J.; Bauer, J.M.; Rämsch, C.; Uter, W.; Guigoz, Y.; Cederholm, T.; Thomas, D.R.; Anthony, P.S.; Charlton, K.E.; Maggio, M.; et al. Frequency of malnutrition in older adults: A multinational perspective using the mini nutritional assessment. J. Am. Geriatr. Soc. 2010, 58, 1734-1738. [CrossRef]

10. Donini, L.M.; Poggiogalle, E.; Molfino, A.; Rosano, A.; Lenzi, A.; Rossi Fanelli, F.; Muscaritoli, M. Mini-nutritional assessment, malnutrition universal screening tool, and nutrition risk screening tool for the nutritional evaluation of older nursing home residents. J. Am. Med. Dir. Assoc. 2016, 17, 959.e11-959.e18. [CrossRef]

11. Kondrup, J.; Allison, S.P.; Elia, M.; Vellas, B.; Plauth, M. ESPEN guidelines for nutrition screening. Clin. Nutr. 2003, 22, 415-421. [CrossRef]

12. Correia, M.I.T.D.; Waitzberg, D.L. The impact of malnutrition on morbidity, mortality, length of hospital stay and costs evaluated through a multivariate model analysis. Clin. Nutr. 2003, 22, 235-239. [CrossRef] 
13. Maseda, A.; Diego-Diez, C.; Lorenzo-López, L.; López-López, R.; Regueiro-Folgueira, L.; Millán-Calenti, J.C. Quality of life, functional impairment and social factors as determinants of nutritional status in older adults: The VERISAÚDE study. Clin. Nutr. 2018, 37, 993-999. [CrossRef] [PubMed]

14. Donini, L.M.; Scardella, P.; Piombo, L.; Neri, B.; Asprino, R.; Proietti, A.R.; Carcaterra, S.; Cava, E.; Cataldi, S.; Cucinotta, D.; et al. Malnutrition in elderly: Social and economic determinants. J. Nutr. Health Aging 2013, 17, 9-15. [CrossRef] [PubMed]

15. Quality Assessment Tool for Observational Cohort and Cross-Sectional Studies. Available online: https: //www.nhlbi.nih.gov/health-topics/study-quality-assessment-tools (accessed on 17 April 2019).

16. Chen, C.C.-H.; Bai, Y.-Y.; Huang, G.-H.; Tang, S.T. Revisiting the concept of malnutrition in older people. J. Clin. Nurs. 2007, 16, 2015-2026. [CrossRef]

17. Boulos, C.; Salameh, P.; Barberger-Gateau, P. Social isolation and risk for malnutrition among older people. Geriatr. Gerontol. Int. 2017, 17, 286-294. [CrossRef]

18. Cabrera, M.A.S.; Mesas, A.E.; Garcia, A.R.L.; de Andrade, S.M. Malnutrition and depression among community-dwelling elderly people. J. Am. Med. Dir. Assoc. 2007, 8, 582-584. [CrossRef]

19. Bardon, L.A.; Streicher, M.; Corish, C.A.; Clarke, M.; Power, L.C.; Kenny, R.A.; O'Connor, D.M.; Laird, E.; O'Connor, E.M.; Visser, M.; et al. Predictors of incident malnutrition in older Irish adults from the Irish longitudinal study on ageing cohort-A MaNuEL study. J. Gerontol. Ser. A 2020, 75, 249-256. [CrossRef]

20. El Zoghbi, M.; Boulos, C.; Awada, S.; Rachidi, S.; Al-Hajje, A.; Bawab, W.; Saleh, N.; Salameh, P. Prevalence of malnutrition and its correlates in older adults living in long stay institutions situated in Beirut, Lebanon. J. Res. Health Sci. 2014, 14, 11-17.

21. Eskelinen, K.; Hartikainen, S.; Nykänen, I. Is loneliness associated with malnutrition in older people? Int. J. Gerontol. 2016, 10, 43-45. [CrossRef]

22. Ferra, A.; Del Mar Bibiloni, M.; Zapata, M.E.; Pich, J.; Pons, A.; Tur, J.A. Body mass index, life-style, and healthy status in free living elderly people in menorca island. J. Nutr. Health Aging 2012, 16, 298-305. [CrossRef]

23. Fjell, A.; Cronfalk, B.S.; Carstens, N.; Rongve, A.; Kvinge, L.M.R.; Seiger, Å.; Skaug, K.; Boström, A.-M. Risk assessment during preventive home visits among older people. J. Multidiscip. Healthc. 2018, 11, 609-620. [CrossRef]

24. Madeira, T.; Peixoto-Plácido, C.; Sousa-Santos, N.; Santos, O.; Alarcão, V.; Goulão, B.; Mendonça, N.; Nicola, P.J.; Yngve, A.; Bye, A.; et al. Malnutrition among older adults living in Portuguese nursing homes: The PEN-3S study. Public Health Nutr. 2019, 22, 486-497. [CrossRef]

25. Mitri, R.; Boulos, C.; Adib, S.M. Determinants of the nutritional status of older adults in urban Lebanon. Geriatr. Gerontol. Int. 2017, 17, 424-432. [CrossRef] [PubMed]

26. Farre, T.B.; Formiga, F.; Ferrer, A.; Plana-Ripoll, O.; Almeda, J.; Pujol, R. Risk of being undernourished in a cohort of community-dwelling 85-year-olds: The Octabaix study. Geriatr. Gerontol. Int. 2014, 14, 702-709. [CrossRef] [PubMed]

27. Park, M.; Kim, H.; Kim, S.K. Knowledge discovery in a community data set: Malnutrition among the elderly. Healthc. Inform. Res. 2014, 20,30-38. [CrossRef] [PubMed]

28. Mokhber, N.; Majdi, M.; Ali-Abadi, M.; Shakeri, M.; Kimiagar, M.; Salek, R.; Moghaddam, P.A.; Sakhdari, A.; Azimi-Nezhad, M.; Ghayour-Mobarhan, M.; et al. Association between malnutrition and depression in elderly people in Razavi Khorasan: A population based-study in Iran. Iran. J. Public Health 2011, 40, 67-74. [PubMed]

29. Damayanthi, H.D.W.T.; Moy, F.M.; Abdullah, K.L.; Dharmaratne, S.D. Prevalence of malnutrition and associated factors among community-dwelling older persons in Sri Lanka: A cross-sectional study. BMC Geriatr. 2018, 18, 199. [CrossRef]

30. Shi, R.; Duan, J.; Deng, Y.; Tu, Q.; Cao, Y.; Zhang, M.; Zhu, Q.; Lü, Y. Nutritional status of an elderly population in Southwest China: A cross-sectional study based on comprehensive geriatric assessment. J. Nutr. Health Aging 2015, 19, 26-32. [CrossRef]

31. Romero-Ortuno, R.; Casey, A.-M.; Cunningham, C.U.; Squires, S.; Prendergast, D.; Kenny, R.A.; Lawlor, B.A. Psychosocial and functional correlates of nutrition among community-dwelling older adults in Ireland. $J$. Nutr. Health Aging 2011, 15, 527-531. [CrossRef] 
32. Rodríguez-Tadeo, A.; Wall-Medrano, A.; Gaytan-Vidaña, M.E.; Campos, A.; Ornelas-Contreras, M.; Novelo-Huerta, H.I. Malnutrition risk factors among the elderly from the US-Mexico border: The "one thousand" study. J. Nutr. Health Aging 2012, 16, 426-431. [CrossRef]

33. Schilp, J.; Wijnhoven, H.A.H.; Deeg, D.J.H.; Visser, M. Early determinants for the development of undernutrition in an older general population: Longitudinal aging study Amsterdam. Br. J. Nutr. 2011, 106, 708-717. [CrossRef]

34. Söderhamn, U.; Dale, B.; Sundsli, K.; Söderhamn, O. Nutritional screening of older home-dwelling Norwegians: A comparison between two instruments. Clin. Interv. Aging 2012, 7, 383-391. [CrossRef]

35. Timpini, A.; Facchi, E.; Cossi, S.; Ghisla, M.K.; Romanelli, G.; Marengoni, A. Self-reported socio-economic status, social, physical and leisure activities and risk for malnutrition in late life: A cross-sectional population-based study. J. Nutr. Health Aging 2011, 15, 233-238. [CrossRef]

36. Suzana, S.; Boon, P.C.; Chan, P.P.; Normah, C.D. Malnutrition risk and its association with appetite, functional and psychosocial status among elderly Malays in an agricultural settlement. Malays. J. Nutr. 2013, 19, 65-75.

37. Ji, L.; Meng, H.; Dong, B. Factors associated with poor nutritional status among the oldest-old. Clin. Nutr. 2012, 31, 922-926. [CrossRef]

38. Westergren, A.; Hagell, P.; Sjödahl Hammarlund, C. Malnutrition and risk of falling among elderly without home-help service-A cross sectional study. J. Nutr. Health Aging 2014, 18, 905-911. [CrossRef]

39. Krzymińska-Siemaszko, R.; Mossakowska, M.; Skalska, A.; Klich-Rączka, A.; Tobis, S.; Szybalska, A.; Cylkowska-Nowak, M.; Olszanecka-Glinianowicz, M.; Chudek, J.; Wieczorowska-Tobis, K. Social and economic correlates of malnutrition in Polish elderly population: The results of PolSenior study. J. Nutr. Health Aging 2015, 19, 397-402. [CrossRef]

40. Johansson, L.; Sidenvall, B.; Malmberg, B.; Christensson, L. Who will become malnourished? A prospective study of factors associated with malnutrition in older persons living at home. J. Nutr. Health Aging 2009, 13, 855-861. [CrossRef]

41. Ferdous, T.; Kabir, Z.N.; Wahlin, Å.; Streatfield, K.; Cederholm, T. The multidimensional background of malnutrition among rural older individuals in Bangladesh-A challenge for the millennium development goal. Public Health Nutr. 2009, 12, 2270-2278. [CrossRef]

42. Mathew, A.C.; Das, D.; Sampath, S.; Vijayakumar, M.; Ramakrishnan, N.; Ravishankar, S. Prevalence and correlates of malnutrition among elderly in an urban area in Coimbatore. Indian J. Public Health 2016, 60, 112-117. [CrossRef]

43. Naidoo, I.; Charlton, K.E.; Esterhuizen, T.M.; Cassim, B. High risk of malnutrition associated with depressive symptoms in older South Africans living in KwaZulu-Natal, South Africa: A cross-sectional survey. J. Health. Popul. Nutr. 2015, 33, 19. [CrossRef]

44. Debnath, S.C. Relationship of socio-economic status with nutritional status among the elderly in a rural community of Bangladesh. Fam. Med. Prim. Care Rev. 2017, 2, 104-109. [CrossRef]

45. El-Desouky, R.; Abed, H. Screening of malnutrition and its correlates among a sample of rural elderly in Qalyobeya Governorate, Egypt. J. Egypt. Public Health Assoc. 2017, 92, 156-166. [CrossRef]

46. Jésus, P.; Guerchet, M.; Pilleron, S.; Fayemendy, P.; Maxime Mouanga, A.; Mbelesso, P.; Preux, P.M.; Desport, J.C. Undernutrition and obesity among elderly people living in two cities of developing countries: Prevalence and associated factors in the EDAC study. Clin. Nutr. ESPEN 2017, 21, 40-50. [CrossRef] [PubMed]

47. Olayiwola, I.O.; Ketiku, A.O. Socio-demographic and nutritional assessment of the elderly Yorubas in Nigeria. Asia Pac. J. Clin. Nutr. 2006, 15, 95-101. [PubMed]

48. Wham, C.; Maxted, E.; Teh, R.; Kerse, N. Factors associated with nutrition risk in older Māori: A cross sectional study. N. Z. Med. J. 2015, 128, 45-54.

49. Ramage-Morin, P.L.; Garriguet, D. Nutritional risk among older Canadians. Heal. Rep. 2013, 24, 3-13.

50. Lengyel, C.O.; Jiang, D.; Tate, R.B. Trajectories of nutritional risk: The Manitoba follow-up study. J. Nutr. Health Aging 2017, 21, 604-609. [CrossRef]

51. Jun, T.; Yuan, Z. Cross sectional study of nutritional status in older han women. Southeast Asian J. Trop. Med. Public Health 2016, 47, 92-100.

52. Gündüz, E.; Eskin, F.; Gündüz, M.; Bentli, R.; Zengin, Y.; Dursun, R.; İçer, M.; Durgun, H.M.; Gürbüz, H.; Ekinci, M.; et al. Malnutrition in community-dwelling elderly in Turkey: A multicenter, cross-sectional study. Med. Sci. Monit. 2015, 21, 2750-2756. [CrossRef] 
53. Lin, W.-Q.; Wang, H.H.X.; Yuan, L.-X.; Li, B.; Jing, M.-J.; Luo, J.-L.; Tang, J.; Ye, B.-K.; Wang, P.-X. The unhealthy lifestyle factors associated with an increased risk of poor nutrition among the elderly population in China. J. Nutr. Health Aging 2017, 21, 943-953. [CrossRef]

54. Programa de las Naciones Unidas para el Desarrollo Índices e Indicadores de Desarrollo Humano. Available online: http://hdr.undp.org/sites/default/files/2018_human_development_statistical_update_es.pdf (accessed on 17 May 2019).

55. Damião, R.; Santos, Á.d.S.; Matijasevich, A.; Menezes, P.R. Factors associated with risk of malnutrition in the elderly in south-eastern Brazil. Rev. Bras. Epidemiol. 2017, 20, 598-610.

56. García-Lorda, P.; Foz, M.; Salas-Salvadó, J. Nutritional status of the elderly population of Catalonia, Spain. Med. Clin. 2002, 118, 707-715. [CrossRef]

57. De Groot, C.P.; van Staveren, W.A.; de Graaf, C. Determinants of macronutrient intake in elderly people. Eur. J. Clin. Nutr. 2000, 54, S70-S76. [CrossRef] [PubMed]

58. Sampson, G. Weight loss and malnutrition in the elderly-the shared role of GPs and APDs. Aust. Fam. Physician 2009, 38, 507-510.

59. McCormack, P. Undernutrition in the elderly population living at home in the community: A review of the literature. J. Adv. Nurs. 1997, 26, 856-863. [CrossRef]

60. Locher, J.L.; Robinson, C.O.; Roth, D.L.; Ritchie, C.S.; Burgio, K.L. The effect of the presence of others on caloric intake in homebound older adults. J. Gerontol. A Biol. Sci. Med. Sci. 2005, 60, 1475-1478. [CrossRef]

61. O'Keeffe, M.; Kelly, M.; O’Herlihy, E.; O’Toole, P.W.; Kearney, P.M.; Timmons, S.; O'Shea, E.; Stanton, C.; Hickson, M.; Rolland, Y.; et al. Potentially modifiable determinants of malnutrition in older adults: A systematic review. Clin. Nutr. 2018, 38, 2477-2498. [CrossRef]

62. Brownie, S. Why are elderly individuals at risk of nutritional deficiency? Int. J. Nurs. Pract. 2006, 12, 110-118. [CrossRef]

63. Shahar, D.R.; Schultz, R.; Shahar, A.; Wing, R.R. The effect of widowhood on weight change, dietary intake, and eating behavior in the elderly population. J. Aging Health 2001, 13, 186-199. [CrossRef]

64. Koo, Y.X.; Kang, M.L.; Auyong, A.; Liau, G.Z.; Hoe, J.; Long, M.; Koh, A.; Koh, F.; Liu, R.; Koh, G. Malnutrition in older adults on financial assistance in an urban Asian country: A mixed methods study. Public Health Nutr. 2014, 17, 2834-2843. [CrossRef]

65. Guyonnet, S.; Rolland, Y. Screening for malnutrition in older people. Clin. Geriatr. Med. 2015, 31, $429-437$. [CrossRef] [PubMed]

66. Payette, H.; Shatenstein, B. Determinants of healthy eating in community-dwelling elderly people. Can. J. Public Health 2005, 96, S27-S31. [PubMed]

67. Chen, S.-H.; Cheng, H.-Y.; Chuang, Y.-H.; Shao, J.-H. Nutritional status and its health-related factors among older adults in rural and urban areas. J. Adv. Nurs. 2015, 71, 42-53. [CrossRef] [PubMed]

68. Grammatikopoulou, M.G.; Gkiouras, K.; Theodoridis, X.; Tsisimiri, M.; Markaki, A.G.; Chourdakis, M.; Goulis, D.G. Food insecurity increases the risk of malnutrition among community-dwelling older adults. Maturitas 2019, 119, 8-13. [CrossRef] [PubMed]

69. Mueller, M.; D’Addario, M.; Egger, M.; Cevallos, M.; Dekkers, O.; Mugglin, C.; Scott, P. Methods to systematically review and meta-analyse observational studies: A systematic scoping review of recommendations. BMC Med. Res. Methodol. 2018, 18, 44. [CrossRef]

70. Higgins, J.P.T.; Thompson, S.G.; Deeks, J.J.; Altman, D.G. Measuring inconsistency in meta-analyses. BMJ 2003, 327, 557-560. [CrossRef]

71. Antentas, J.M.; Vivas, E. Impacto de la crisis en el derecho a una alimentación sana y saludable. Informe SESPAS 2014. Gac. Sanit. 2014, 28, 58-61. [CrossRef]

72. Volkert, D.; Beck, A.M.; Cederholm, T.; Cruz-Jentoft, A.; Goisser, S.; Hooper, L.; Kiesswetter, E.; Maggio, M.; Raynaud-Simon, A.; Sieber, C.C.; et al. ESPEN guideline on clinical nutrition and hydration in geriatrics. Clin. Nutr. 2019, 38, 10-47. [CrossRef]

(C) 2020 by the authors. Licensee MDPI, Basel, Switzerland. This article is an open access article distributed under the terms and conditions of the Creative Commons Attribution (CC BY) license (http://creativecommons.org/licenses/by/4.0/). 